\title{
Anisotropy in the magnetic and electrical transport properties of $\mathrm{Fe}_{1-x} \mathrm{Cr}_{x} \mathrm{Sb}_{2}$
}

\author{
Rongwei $\mathrm{Hu},{ }^{1,2}$ V. F. Mitrović, ${ }^{2}$ and C. Petrovic ${ }^{1}$ \\ ${ }^{1}$ Condensed Matter Physics and Materials Science Department, \\ Brookhaven National Laboratory, Upton, NY 11973 \\ ${ }^{2}$ Department of Physics, Brown University, Providence, RI 02912
}

(Dated: December 1, 2018)

\begin{abstract}
We have investigated anisotropy in magnetic and electrical transport properties of $\mathrm{Fe}_{1-x} \mathrm{Cr}_{x} \mathrm{Sb}_{2}$ $(0 \leq x \leq 1)$ single crystals. The magnetic ground state of the system evolves from paramagnetic to antiferromagnetic with gradual substitution of Fe with Cr. Anisotropy in electrical transport diminishes with increased Cr substitution and fades away by $x=0.5$. We find that the variable range hopping $(\mathrm{VRH})$ conduction mechanism dominates at low temperatures for $0.4 \leq x \leq 0.75$.
\end{abstract}

PACS numbers: 71.20.Nr, 71.28.+d, 75.30.-m, 75.50.Pp

\section{INTRODUCTION}

The magnetic resemblance of $\mathrm{FeSb}_{2}$ to FeSi suggests that $\mathrm{FeSb}_{2}$ could be another model system to study the possible applicability of the Kondo insulator scenario to transition metal compounds with $3 d$ electrons $, \underline{1}, \underline{2}, \underline{3}, \underline{\underline{4}}$ The magnetic susceptibility of $\mathrm{FeSb}_{2}$ is nearly temperature independent at low temperatures, followed by diamagnetic to paramagnetic crossover around $100 \mathrm{~K}$ and thermally activated behavior at high temperatures $\underline{\underline{5}}$ Another striking similarity to FeSi comes from the result of ab initio $\mathrm{LDA}+U$ electronic calculations that show near degeneracy of small gap semiconductor and metallic ferromagnetic state $\underline{\underline{4}}$ Electrical transport in $\mathrm{FeSb}_{2}$ is rather anisotropic and nearly quasi one-dimensional. Whereas resistivity along the $\widehat{a}^{-}$and $\widehat{c}^{-}$axes is semiconducting showing activated temperature dependence below room temperature, the $\widehat{b}$ - axis resistivity is metallic above 40 $\mathrm{K}$ and semiconducting below that temperature $\stackrel{1}{*}$ Optical conductivity measurements showed a true insulating state, zero Drude weight of $\sigma(\omega)$ at low frequencies and an anisotropic energy gap $\mathrm{E}_{g}$ in the spectral range between $100-350 \mathrm{~cm}^{-1}$ in overall agreement with resistivity measurements. Thermal excitations of charge carriers through $\mathrm{E}_{g}$ in conventional MIT transitions produce redistribution of the spectral weight just above the gap. However, a full recovery of spectral weight in $\mathrm{FeSb}_{2}$ occurs above $1 \mathrm{eV}$, suggesting contributions of larger energy scales ${ }^{\underline{3}}$ Electron doping on Fe site induced a weak ferromagnetic metallic state and colossal magnetoresistance in $\mathrm{Fe}_{1-x} \mathrm{Co}_{x} \mathrm{Sb}_{2}$. Thus other doping studies in this system are of particular interest. $\underline{\underline{5}}, \underline{6}$

Hole doping, on the other hand, induces a heavy fermion metallic state in $\mathrm{FeSb}_{2-x} \mathrm{Sn}_{x} \frac{7}{\underline{7}}$, analogous to the

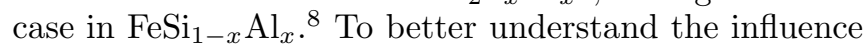
of the hole doping on the ground state of $\mathrm{FeSb}_{2}$, we investigate the effects of $\mathrm{Cr}$ substitution on Fe site of this material. The significance of $\mathrm{Cr}$ substitution is that holes are introduced in narrow energy bands with $3 d$ character, unlike in the case of $\mathrm{Co}$ and $\mathrm{Sn}$ substitution. $\mathrm{CrSb}_{2}$ is an antiferromagnetic semiconductor that crystallizes into the marcasite structure with the same space group
Pnnm .9 Neutron diffraction studies of $\mathrm{CrSb}_{2}$ indicate a high spin $d^{2}$ configuration, an antiferromagnetic ground state with Neel temperature $\mathrm{T}_{N}=273 \pm 2 \mathrm{~K}$, and a magnetic moment per $\mathrm{Cr}$ of $1.94 \mu_{B}, \frac{10}{=} \mathrm{In}$ this work we examine the magnetic and electrical properties of $\mathrm{Fe}_{1-x} \mathrm{Cr}_{x} \mathrm{Sb}_{2}$ $(0 \leq x \leq 1)$ and discuss the possible conduction mechanism of this binary system.
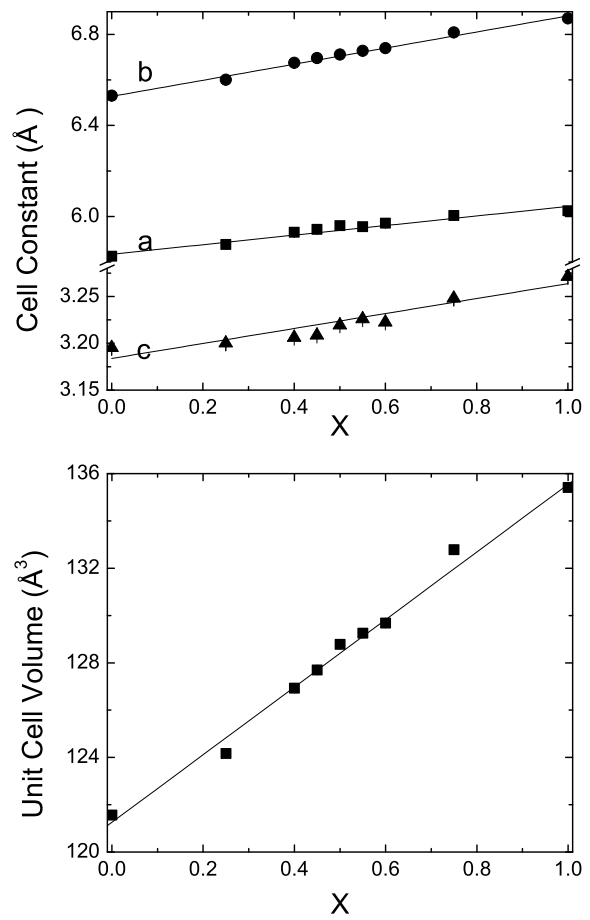

FIG. 1: Lattice constants and unit cell volume of $\mathrm{Fe}_{1-x} \mathrm{Cr}_{x} \mathrm{Sb}_{2}$ versus nominal $\mathrm{Cr}$ concentration $x$. 


\section{EXPERIMENTAL METHOD}

The samples investigated in our experiments were single crystals grown from high temperature melt 11,12 Powder X-ray diffraction (XRD) spectra of the ground samples were taken with $\mathrm{Cu} \mathrm{K}_{\alpha}$ radiation $(\lambda=1.5418 \AA)$ using a Rigaku Miniflex X-ray machine. The lattice parameters were obtained by fitting the XRD spectra using the Rietica software 13 Single crystals were oriented using a Laue Camera and were polished into rectangular bars along specific crystalline axes. Thin Pt wires were attached to electrical contacts made with Epotek H20E silver epoxy for a standard 4-wire resistance measurement. Sample dimensions were measured with an optical microscope Nikon SMZ-800 with $10 \mu \mathrm{m}$ resolution, and the average values were used to estimate a geometric factor. Magnetization, resistivity and heat capacity measurements were carried out in a Quantum Design MPMS5 and a PPMS-9 for temperatures from $1.8 \mathrm{~K}$ to $350 \mathrm{~K}$.

\section{RESULTS AND DISCUSSION}

The lattice parameters of doped samples from the powder X-ray diffraction spectra are shown in Fig. 1. Linear dependence on $\mathrm{Cr}$ concentration in accordance with Vegard's law demonstrates that $\mathrm{Cr}$ uniformly substitutes Fe in the entire doping range. All axes increase linearly with increasing $x$ and the lattice constants of the end members are consistent with the reported values, FeSb 2 $(a=5.8253(2) \AA, b=6.5313(2) \AA, c=3.1952(2) \AA)$ and $\mathrm{CrSb}_{2}(a=6.0250(1) \AA, b=6.8708(1) \AA, c=3.2711(1)$ A) $1,14,16$

The magnetic susceptibility along the three crystal axes of $\mathrm{Fe}_{1-x} \mathrm{Cr}_{x} \mathrm{Sb}_{2}(0 \leq x \leq 1)$ in temperature range 1.8 to $350 \mathrm{~K}$ is shown in Fig. 2. FeSb 2 exhibits a diamagnetic to paramagnetic crossover at about $100 \mathrm{~K}$ for field applied along the $\widehat{c}$ axis. The temperature dependence of the susceptibility along the $\widehat{a}$ - and $\widehat{b}$ - axes is virtually identical: nearly temperature independent at low temperatures and thermally activated at higher temperatures. The magnetic susceptibility of $\mathrm{CrSb}_{2}$ is consistent with magnetic ordering below $\mathrm{T}_{N}=275 \mathrm{~K}$. A free ion model Eq.(1) proposed by Jaccarino applied to $\mathrm{FeSb}_{2}$ gives a spin gap $\Delta_{\chi}=527 \pm 4 K{ }^{17}$

$$
\begin{aligned}
\chi_{F I} & =N g^{2} \mu_{B}^{2} \frac{J(J+1)}{3 k_{B} T} \frac{2 J+1}{2 J+1+\exp \left(\Delta_{\chi} / k_{B} T\right)}+\chi_{0} \\
\chi_{C W} & =\frac{N \mu_{e f f}^{2}}{3 k_{B}(T-\Theta)}
\end{aligned}
$$

For $x=0.1$, the temperature dependence of susceptibility is comprised of a reduced thermally activated term, Eq.(1), with gap value $\Delta_{\chi}=441 \pm 8 K$, and a low-temperature Curie-Weiss term, Eq.(2), with $\mu_{e f f}=$ $1.08 \pm 0.06 \mu_{B}$ due to $\mathrm{Cr}$ substitution. With further $\mathrm{Cr}$
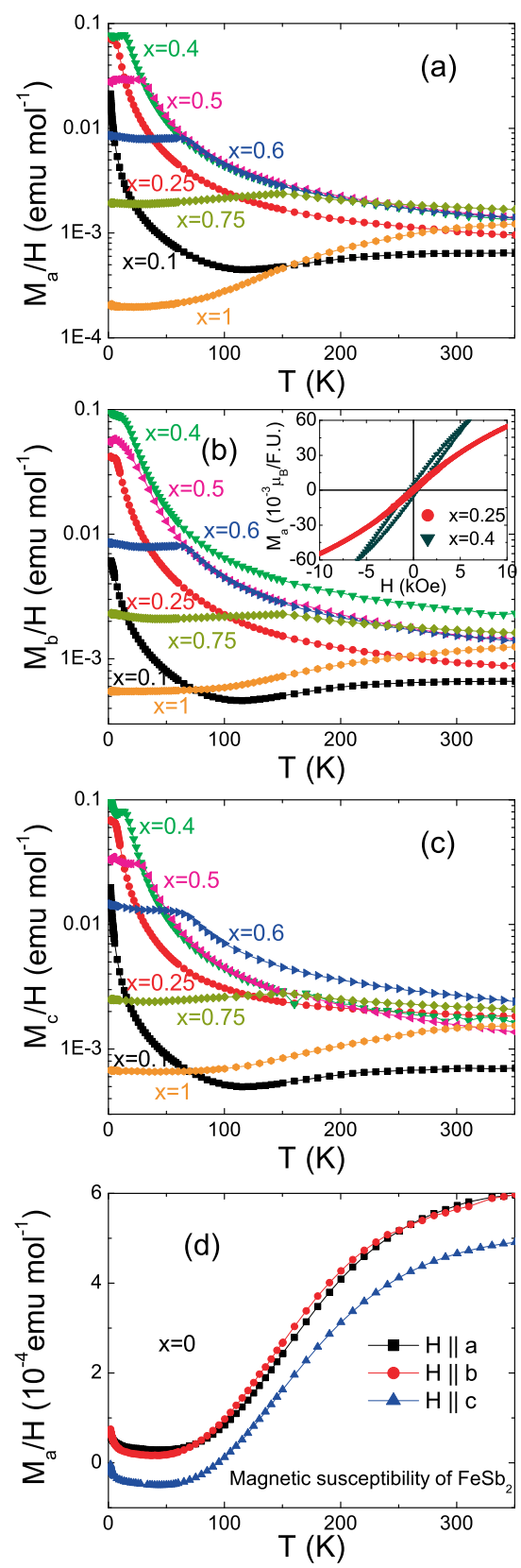

FIG. 2: (a)-(c)Temperature dependance of $M / H$ for field of $H=1000$ Oe applied along 3 crystalline axes. Inset shows the hysteresis loops for $0.25 \leq x \leq 0.4$. (d) $M / H$ versus temperature $(T)$ of pure $\mathrm{FeSb}_{2}$.

substitution, long range antiferromagnetic order is induced for $x \geq 0.25$ and the Neel temperature gradually increases toward $\mathrm{T}_{N}=275 \mathrm{~K}$, of $\mathrm{CrSb}_{2}(x=1)$. The high temperature effective magnetic moment is somewhat lower than expected, $\mu_{\text {eff }}\left(\mathrm{Cr}^{4+}\right)=2 \mu_{B}$ for the end concentrations of $x=0.1$ and $x=0.75$, as summarized in Table I. This deviation could arise due to contributions of the thermally activated $\mathrm{FeSb}_{2}$-like Pauli susceptibility 
TABLE I: Parameters of the fits to the Curie-Weiss law of the high temperature $M / H$ and semiconducting energy gaps of the resistivity.

\begin{tabular}{|c|c|c|c|c|c|c|}
\hline$x$ & $\mu_{e f f}\left(\mu_{B}\right)$ & $\Theta(K)$ & $\chi_{0}(\mathrm{emu} / \mathrm{mol})$ & $T_{C} / T_{N}(K)$ & $M s\left(10^{-3} \mu_{B}\right)$ & $\Delta_{\rho}(K)$ \\
\hline 0.10 & $1.08(6)$ & $22(8)$ & $1.3 \times 10^{-4}$ & & & $302(9)$ \\
\hline 0.25 & $1.13(5)$ & $26(4)$ & $8.2 \times 10^{-4}$ & $6.6(5)$ & $45(1)$ & $321(2)$ \\
\hline 0.40 & $1.85(6)$ & $18(3)$ & $3.8 \times 10^{-4}$ & $14(1)$ & $72(1)$ & $434(8)$ \\
\hline 0.50 & $1.88(2)$ & $2.5(8)$ & $2.2 \times 10^{-4}$ & 25 & & $425(1)$ \\
\hline 0.60 & $1.82(4)$ & $-12(3)$ & $6.3 \times 10^{-4}$ & $65(2)$ & & $397(6)$ \\
\hline 0.75 & $1.44(3)$ & $-18(1)$ & $12.2 \times 10^{-4}$ & $150(5)$ & & $251(4)$ \\
\hline
\end{tabular}

for low Cr doping and to temperature limitations of our magnetic susceptibility measurement, where $\chi(T)$ curves were fitted in close proximity to the antiferromagnetic transition for $x=0.75$.

Parameters deduced from the fit to the Eq. (1) model are listed in Table I. Clearly, the Curie-Weiss temperature changes sign from positive to negative indicating that the magnetic coupling evolves from ferromagnetic to antiferromagnetic. However, the field dependence of the transition, as depicted in Fig. 3, reveals a canted antiferromagnetic structure for $0.25 \leq x \leq 0.45$. In this doping range, in a predominantly antiferromagnetic phase a small spontaneous magnetization is present due to a slight deviation from a strictly antiparallel arrangement or to parasitic ferromagnetism. Since there is an inversion symmetry at the Fe site in the Pnnm space group of $\mathrm{FeSb}_{2}$, we can exclude the presence of DzayloshinskyMoriya type of interaction. ${ }^{18}$ The seemingly ferromagnetic tail is sensitive to the applied field, changing to a characteristic antiferromagnetic peak in lower fields. Magnetism in $\mathrm{Fe}_{1-x} \mathrm{Cr}_{x} \mathrm{Sb}_{2}$ is most likely attributable to the ordering of $\mathrm{Cr}$ atoms since the electronic configuration of $\mathrm{Fe}$ in $\mathrm{FeSb}_{2}$ is the nonmagnetic $3 d^{4}$. Assum-
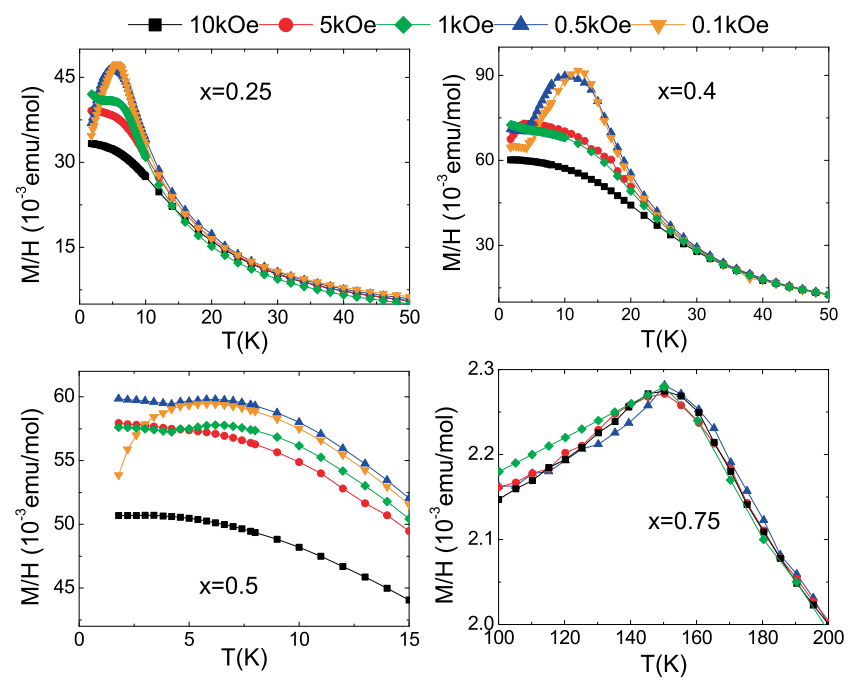

FIG. 3: Field dependance of the magnetic transitions for $0.25 \leq x \leq 0.75$ ing that the magnetization $M(H)$ consists of a saturation moment $\left(M_{s}\right)$ and paramagnetic component $(\chi H)$, we obtain the canting angle $\phi$ of $2.2 \sim 4.1^{\circ}$. The angle is defined as $2 \sin ^{-1}\left(M_{S} / 2 M_{\max }\right)$ where $M_{\max }=2 \mu_{B}$ is the magnetic moment of the $\mathrm{Cr}^{4+}$. The saturation moment $M_{S} \sim 0.09 \mu_{B} / \mathrm{Cr}$ accounts for the ordering of $4.5 \%$ of $\mathrm{Cr}$ atoms. Hysteresis loops with coercive fields of $H_{C}=(230 \sim 1100) O e$ and remnant fields of $H_{R}=(10 \sim 63)$ Oe emu/mol were observed with field applied along all three crystalline axes in this doping range. With further $\mathrm{Cr}$ substitution for $0.6 \leq x \leq 1$, the system has the antiferromagnetic structure of $\mathrm{CrSb}_{2}$, consistent with the results of Kjekshus et al. 14

The temperature dependence of the resistivity of $\mathrm{Fe}_{1-x} \mathrm{Cr}_{x} \mathrm{Sb}_{2}$ for current applied along individual axes of the crystal structure is shown in Fig. 4. Electrical transport along the $\widehat{b}$ - axis shows a substantial metallic region for $0 \leq x \leq 0.25$. The region of metallic resistivity gradually shifts down in temperature with increasing $x$, yielding a metallic state at the lowest measured temperature for $x=0.25$. At high temperatures, however, the region of metallic resistivity is progressively reduced with the increasing $x$, giving way to semiconducting behavior above $150 \mathrm{~K}$. Above $150 \mathrm{~K}$ activated temperature dependence of resistivity is isotropic for all $x \geq 0.1$, except for $\rho_{b}$ of $x=0.75$.

The evolution of the intrinsic band semiconducting energy gap is listed in Table I. The gap values increase in nonmonotonic fashion from $\Delta_{\rho}=225 \pm 5 K$ for $x=0$ to $\Delta_{\rho}=834 \pm 3 K$ for $x=1$. For $x=0.4$, the gap value reaches its local maximum. Semiconducting energy gap for $x=0.75$ deviates from monotonic behavior possibly due to semimetallic transport observed for $\widehat{b}$-axis resistivity at high temperatures. There is a loss of spin disorder scattering at the $\mathrm{T}_{N}$ for $0.25 \leq x \leq 0.1$, in good agreement with magnetization measurements.

The resistivity data for an intermediate range of $x$, $0.25 \leq x \leq 0.75$, is depicted in Fig. 5. Low temperature resistivity is described by $\rho=\rho_{0} \exp \left(T_{0} / T\right)^{1 / 4}$, implying a variable range hopping $(\mathrm{VRH})$ conduction mechanism. The characteristic temperatures $T_{0}$ and the corresponding localization lengths $\xi$ are summarized in Table II. The length is given by $\xi^{3}=19 /\left[k_{B} T_{0} N\left(E_{F}\right)\right]^{15}$, where the density of states at the Fermi level $N\left(E_{F}\right)$ of $3 \times 10^{37} \mathrm{~J}^{-1} \mathrm{~cm}^{-3}$ is estimated using low temperature specific heat measurements (Fig. 5 inset). 

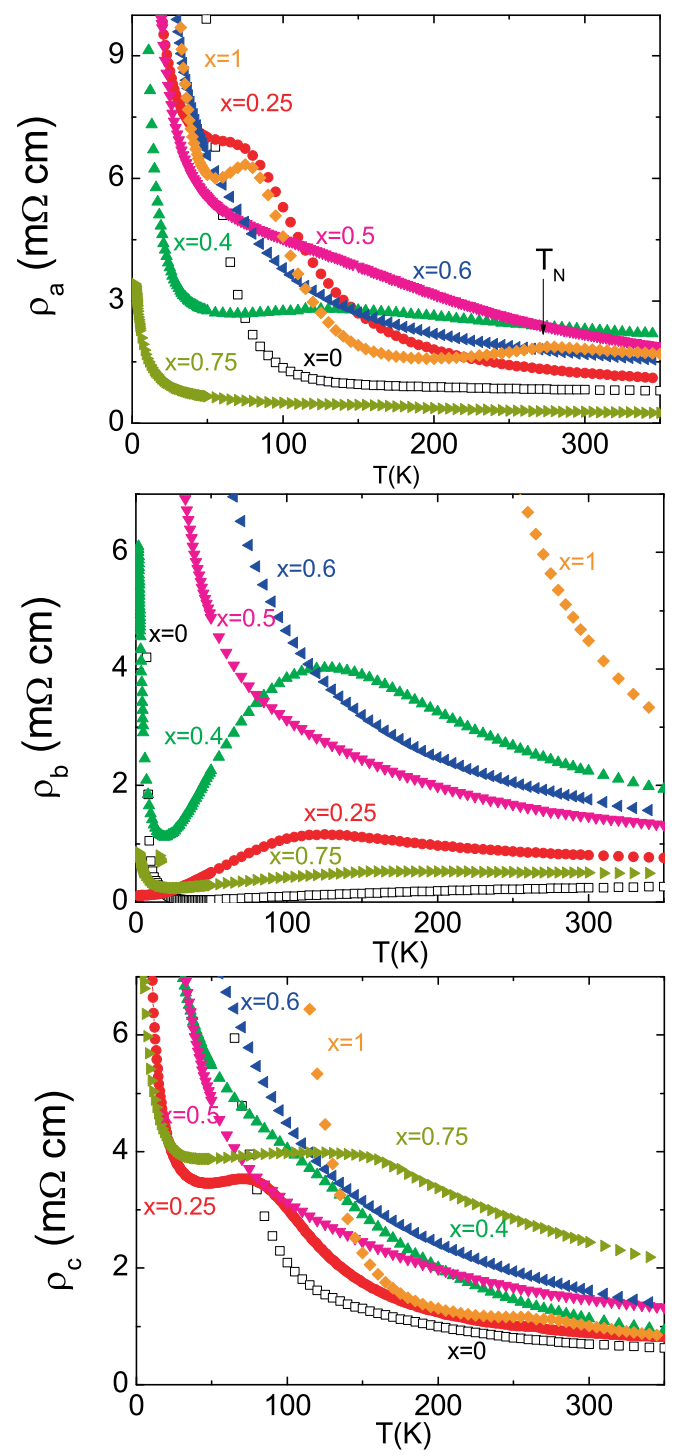

FIG. 4: Resistivity of $\mathrm{Fe}_{1-x} \mathrm{Cr}_{x} \mathrm{Sb}_{2}$ for current applied along three crystalline axes. Arrow indicates the Neel temperatures.

The rich phase diagrams of the electronic system in the $\mathrm{Fe}_{1-x} \mathrm{Cr}_{x} \mathrm{Sb}_{2}$ alloys are displayed in Fig. 6. With the increase of $x$, the paramagnetic ground state evolves toward an antiferromagnetic one, see Fig. 6(a). In the antiferromagnetic part of the phase diagram we distinguish a canted antiferromagnetic (CAF) region for $0.2 \leq x \leq 0.45$. The phase diagram compiled from the electrical transport properties is shown in Fig. 6(b). The $\widehat{b}$ - axis transport is metallic for $0 \leq x \leq 0.25$. In addition, there are two semiconducting regions. The system is band gap semiconductor for $0.25<x$ at high temperature. The variable range hopping (VRH) mechanism

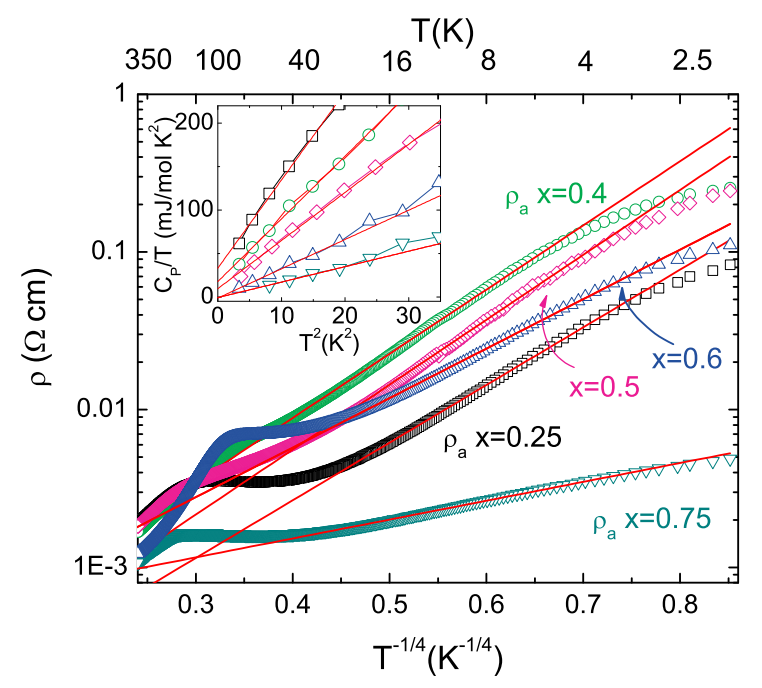

FIG. 5: $\quad \rho$ versus $T^{-1 / 4}$ curves for $0.25 \leq x \leq 0.75$ on a semilog scale. Inset: $\mathrm{C}_{p} / T$ versus $T^{2}$ for $T<6 \overline{\mathrm{K}}$.

is present for intermediate alloys for $0.25 \leq x \leq 0.75$. The low temperature insulating region in $\mathrm{FeSb}_{2}$ diminishes with increasing $x$ in $\mathrm{Fe}_{1-x} \mathrm{Cr}_{x} \mathrm{Sb}_{2}$ and disappears by $x=0.25$.

$\mathrm{FeSb}_{2}$ and $\mathrm{CrSb}_{2}$ crystallize in an orthorhombic marcasite Pnnm structure, whereas $\mathrm{CoSb}_{2}$ has a pseudomarcasite monoclinic $P 21 / c$ symmetry induced by an increase of $\widehat{a}$ - axis, doubling of the $\widehat{c}$ - axis and a small monoclinic angle $\beta=90.4^{\circ}$. $\mathrm{FeSb}_{2}, \mathrm{CoSb}_{2}$ and $\mathrm{CrSb}_{2}$ are all semiconducting materials and continuously miscible on their respective binary alloy phase diagrams. Thus, it is possible to control a rich formation of ground states by carrier concentration. Both $\mathrm{Fe}_{1-x} \mathrm{Co}_{x} \mathrm{Sb}_{2}$ and $\mathrm{Fe}_{1-x} \mathrm{Cr}_{x} \mathrm{Sb}_{2}$ electronic systems are noteworthy in that although the two end members are semiconducting, metallic regions exist in their binary alloy systems. In contrast to the intermediate $\mathrm{Fe}_{1-x} \mathrm{Co}_{x} \mathrm{Sb}_{2}$ system, where a metallic region appears with as small an electron doping as $x=0.05$, in $\mathrm{Fe}_{1-x} \mathrm{Cr}_{x} \mathrm{Sb}_{2}$ ground state metallicity is observed only for $x=0.25$. This is consistent with $\mathrm{LDA}+\mathrm{U}$ band structure calculations $\underline{\underline{4}}$ The density of states (DOS) above the Fermi level is largely determined by the occupancy of $3 d$ orbitals. Electron doping readily moves the Fermi level into the large peak just above the Fermi energy, in contrast to hole doping which moves the Fermi level to the depleted region of the DOS toward the valence band.

TABLE II: $\mathrm{T}_{0}$ and $\xi$ of variable range hopping and Sommerfeld coefficient from specific heat measurements.

\begin{tabular}{llllll}
\hline \hline$x$ & 0.25 & 0.4 & 0.5 & 0.6 & 0.75 \\
\hline$T_{0}(K)$ & 2723 & 4657 & 7063 & 7750 & 57 \\
$\xi(\AA)$ & 215 & 193 & 198 & 254 & 1758 \\
$\gamma\left(\mathrm{mJ} / \mathrm{molK}^{2}\right)$ & 33.1 & 17.7 & 10.7 & 2.1 & 0.1 \\
\hline \hline
\end{tabular}



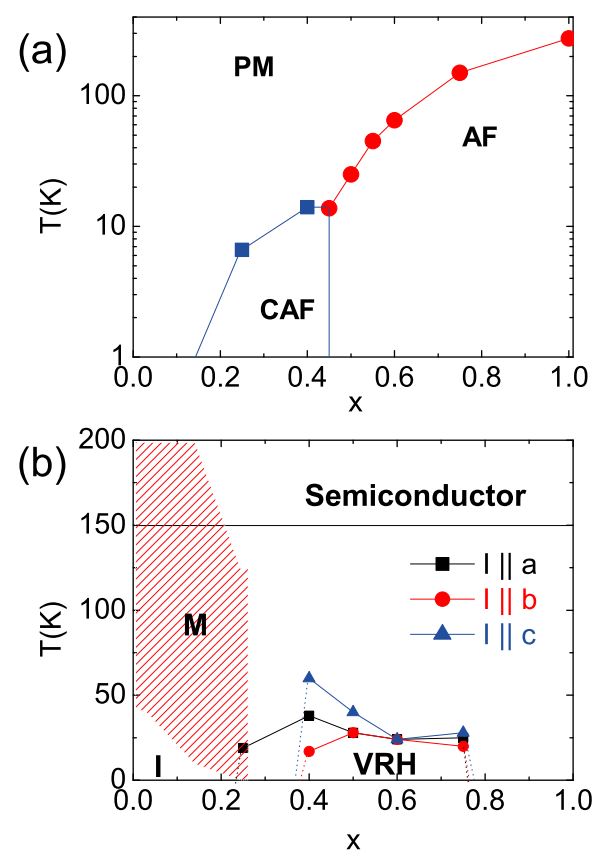

FIG. 6: Phase diagram of $\mathrm{Fe}_{1-x} \mathrm{Cr}_{x} \mathrm{Sb}_{2}$, compiled from measurements of magnetic properties (a) and from electrical transport (b).

\section{CONCLUSION}

We have studied anisotropy in the magnetic and electrical transport properties of $\mathrm{Fe}_{1-x} \mathrm{Cr}_{x} \mathrm{Sb}_{2}$. The substi- tution of $\mathrm{Cr}$ for Fe induced a rich phase diagram. Magnetically ordered ground states are formed for $0.25 \leq$ $x \leq 1$. The canted antiferromagnetism of intermediate alloys for $x \leq 0.5$ evolves into the antiferromagnetic state of $\mathrm{CrSb}_{2}$ with a Neel temperature gradually increasing up to $\mathrm{T}_{N}=275 \mathrm{~K}$ for $x=1$. The material is semiconducting, with a metallic region for $\widehat{b}$ - axis transport in the paramagnetic region for $0 \leq x \leq 0.25$. The isostructural $\mathrm{Fe}_{1-x} \mathrm{Cr}_{x} \mathrm{Sb}_{2}$ alloys are an interesting playground for studying intermediate electronic states between small gap, nearly magnetic (or "Kondo") semiconductor $(x=0)$ and antiferromagnetic semiconductor $(x=1)$. Further optical and X-ray scattering measurements could be useful to study this problem in more detail.

\section{ACKNOWLEDGMENTS}

We thank W.-G. Yin and Myron Strongin for useful communication and critical reading. This work was carried out at the Brookhaven National Laboratory, which is operated for the U.S. Department of Energy by Brookhaven Science Associates (DE-Ac02-98CH10886). This work was supported by the Office of Basic Energy Sciences of the U.S. Department of Energy. This work was also supported in part by the National Science Foundation DMR-0547938 (V. F. M.).
${ }^{1}$ C. Petrovic, J. W. Kim, S. L. Bud'ko, A. I. Goldman and P. C. Canfield, Phys. Rev. B 67, 155205 (2003).

2 C. Petrovic, Y. Lee, T. Vogt, N. Dj. Lazarov, S. L. Bud'ko and P. C. Canfield, Phys. Rev. B 72, 045103 (2005).

3 A. Perucchi, L. Degiorgi, R. Hu, C. Petrovic, and V. Mitrović, European Physical Journal B 54, 175 (2006).

4 A. V. Lukoyanov, V. V. Mazurenko, V. I. Anisimov, M.Sigrist, and T. M. Rice, European Physical Journal B 54(2), 205 (2006).

5 Rongwei Hu, V. F. Mitrovic, and C. Petrovic, Phys. Rev. B 74, 195130 (2006).

6 Rongwei Hu et al., submitted to Phys. Rev. B. (2007).

7 A. Bentien et al, Phys. Rev. B 74, 205105 (2006).

8 J.F.DiTusa, K.Friemelt, E.Bucher, G.Aeppli, A.P.Ramirez, Phys. Rev. B 58, 10288 (1998).

9 J. B. Goodenough, J. Solid State Chem. 5, 144 (1972).

${ }^{10}$ H. Holseth, A. Kjekshus, Acta Chem. Scand. 24, 3309 (1970).

11 P. C. Canfield, Z. Fisk Phil. Magaz. B 65, 1117 (1992).

12 Z. Fisk, J. P. Remeika, in: K. A. Gschneider, J. Eyring
(Eds.), Handbook on the Physics and Chemistry of Rare Earths, Vol. 12, Elsevier, Amsterdam, (1989).

13 Hunter B., "Rietica - A visual Rietveld program", International Union of Crystallography Commission on Powder Diffraction Newsletter No. 20, (Summer) http://www.rietica.org (1998).

14 H. Holseth, A. Kjekshus, Acta Chem. Scand. 223284 (1968).

15 Friedman JR, Zhang YZ, Dai PH, Sarachik MP, Phys. Rev. B 53, 9528 (1996).

16 Harada T., Kanomata T., Takahashi Y., et al, J. Alloys Compounds, 383(1-2) 200, (2004).

17 V. Jaccarino, G. K. Wertheim, J. H. Wernick, L. R. Walker, and S. Arajs, Phys. Rev. 160, 476 (1967).

18 B. Lebech, J. Bernhard and T. Freltoft, J. Phys. Cond. Matter 1, 6105 (1989).

19 A. Kjekshus, P. Petezens, T. Rakke, A.F. Andresen, Acta Chem. Scand. A33 469 (1979). 